\title{
Doença de Chagas como causa básica de óbito na região sudeste do Brasil: presença de causas contributárias
}

\author{
Chagas'disease as principal cause of death an area of southeastern Brazil: presence \\ of contributory causes
}

Dalva Marli Valério Wanderley", Julio Litvoc**

\begin{abstract}
WANDERLEY, D.M.V. \& LITVOC, J. Doença de Chagas como causa básica de óbito na região sudeste do Brasil: presença de causas contributárias. Rev. Saúde Pública, 28: 69-75, 1994. Estudaram-se os atestados de pessoas falecidas no Estado de São Paulo, Brasil, em 1987, cuja causa básica foi a cardiopatia chagásica, com a finalidade de conhecer a informação adicional que está presente no atestado como "causa contributária". Foram analisadas as informações existentes em 1.308 Declarações de Óbito. As causas contributárias foram identificadas e registradas a partir de uma leitura direta do atestado. Foram identificadas 261 Declaraçōes com causas contributárias (20\%), sendo 185 com apenas uma causa registrada e 75 com duas. As seis primeiras causas foram: megas, embolias, doença pulmonar crônica, infecções (exceto doença de Chagas), hipertensão arterial e desnutrição. Analisando a presença das causas contributárias no subgrupo de menores de 50 anos e de 50 anos ou mais, constatou-se uma maior proporção no grupo mais idoso e um perfil diferenciado de causas em cada subgrupo. As causas contributárias não apresentaram associação com sexo e local de residência.
\end{abstract}

Descritores: Tripanossomose sul-americana, mortalidade. Causa da morte.

\section{Introdução}

As informações procedentes do campo "atestado médico" da Declaração de Óbito têm sido analisadas, no Município de São Paulo, a partir de dois procedimentos: privilegiando-se a "causa básica de morte ${ }^{\prime \prime}$, ou utilizando-se de todos os dados registrados no atestado (linhas $a, b, c$ da parte I, e parte II), ou seja, analisando não só a causa básica como também as conseqüências e as contributárias $^{13}$. Para ilustrar esses usos pode-se citar, em relação ao primeiro procedimento, o trabalho de Jatene e col. ${ }^{5}$ e o da Secretaria de Saúde Municipal de São Paulo ${ }^{14}$, que se utilizaram das informações sobre causa básica de óbito na elaboração de um diagnóstico de situação de saúde do município; na outra linha, pode-se citar a pesquisa de Laurenti ${ }^{7}$, que estudou os óbitos ocorridos em hospitais, em

* Superintendência de Controle de Endemias (SUCEN) - São Paulo - SP, Brasil

* Departamento de Medicina Preventiva da Faculdade de Medicina da Universidade de São Paulo - São Paulo, SP Brasil

Separatas/Reprints: D.M.V. Wanderley - Rua Paula Souza, 166 01027-000 - São Paulo, SP - Brasil

Edição subvencionada pela FAPESP. Processo 94/0500-0
1972, e o relatório da Fundação $\operatorname{SEADE}^{3}$, que publicou, em 1987, informações sobre "causas múltiplas" ocorridas em residentes do Estado e Municipio de São Paulo, nos anos de 1983 e 1984.

Embora essas duas abordagens não se constituam em posturas conflitantes, elas contêm perspectivas diversas, que merecem ser ressaltadas: ao se trabalhar com "causa básica" está se privilegiando a noção de "cortar no início a cadeia de eventos" ${ }^{8}$ que conduziram à morte ou que produziram a lesão fatal. É a idéia de "prevenir a ação da causa precipitante" ; já ao se trabalhar com o conjunto de informaçōes do atestado está se obtendo, segundo Laurenti ${ }^{7}$, dois tipos importantes de informações, ou seja, a freqüência total das causas existentes na população falecida e associação de causas.

Esta segunda abordagem coloca também em evidência uma característica importante do processo saúde-doença, que consiste na freqüência com que ocorre a simultaneidade de doenças em uma mesma pessoa; essa simultaneidade é descrita não só nas pessoas falecidas, que são pacientes em geral mais graves, como também nas pessoas atendidas em ambulatórios ou pesquisadas em inquéritos populacionais $^{1,2,12}$.

A partir desse contexto, estudaram-se os atestados de pessoas falecidas no Estado de São Paulo, 
em 1987, cuja causa básica foi a cardiopatia chagásica, com a finalidade de conhecer a informação adicional que está presente nesses atestados como "causa contributária", e contribuir para um melhor conhecimento do perfil de morbi-mortalidade que acomete o grupo populacional com doença de Chagas.

\section{Material e Método}

Em trabalho anterior ${ }^{9}$, foram analisadas as informações existentes em 1.516 Declarações de Óbito de pessoas residentes no Estado de São Paulo, falecidas em 1987, das quais 1.308 apresentaram cardiopatia chagásica como causa básica (086.0; CID-9) ${ }^{11}$. Para identificação da causa básica foram aplicadas as regras de seleção utilizadas internacionalmente ${ }^{11}$. O presente estudo analisa as $1.308 \mathrm{DO}$. Esse material foi obtido a partir de levantamento manual em arquivos da Fundação Sistema Estadual de Análise de Dados (SEADE).

As variáveis analisadas foram: sexo, idade, região onde ocorreu o falecimento e causas contributárias (CC) (parte II do atestado de óbito). Para comparar o comportamento das CC em dois grupos de idade, as 1.308 Declarações foram divididas a partir de um ponto de corte que foi a idade de 50 anos. A decisão sobre esse ponto apoiou-se nas tendências mais recentes dos estudos de assistência ao idoso ${ }^{16}$. Já para examinar a associação entre idade e presença de CC, as 1.308 Declaraçōes foram divididas em 3 subgrupos de idade: até 39 anos, de 40 a 59 e maiores de 60 anos.

As CC foram identificadas e registradas a partir de uma leitura direta do atestado. As categorias nosológicas de classificação destas CC foram elaboradas a partir do material analisado.

Essas categorias foram, em grande parte, doenças isoladas, como é o caso do acidente vascular cerebral (AVC) e da hipertensão arterial, ou conjunto de doenças, como por exemplo as "doenças pulmonares crônicas" ou mesmo as de capitulos da CID, como a Neoplasia (Cap. II); cabe destacar que a rubrica "doenças infecciosas" compreende todo o primeiro capítulo da CID-9, excluída a categoria doença de Chagas (086). Os casos de megacolon, megaesôfago, ou megacolon mais megaesôfago foram agrupados sob a rubrica de "megas".

Além de trabalharmos com o número absoluto de $\mathrm{CC}$, foram calculadas duas proporções: uma primeira, que denominamos de "proporção de presença de causas contributárias", onde o numerador é constituído pelo número de atestados que apresentam, simultaneamente, cardiopatia chagásica como causa básica (na parte I) e causa(s) contributária(s) na parte II; já o denominador é formado pela totalidade dos atestados cuja causa básica foi a cardiopatia chagásica, independente de possuir, ou não, CC. Uma segunda, que se denominou de "proporção de presença de uma causa contributária (específica)", onde o numerador é constituído pelos atestados que apresentam, simultaneamente, cardiopatia chagásica como causa básica e uma CC específica, como por exemplo, o AVC, ou neoplasia, ou doença pulmonar crônica; o denominador, novamente, é formado pela totalidade dos atestados cuja causa básica foi a cardiopatia chagásica, independente de possuir, ou não, CC. Em alguns casos essas proporções foram calculadas para pessoas com menos de 50 anos e de 50 anos ou mais.

As associações foram analisadas comparando proporções por meio da distribuição $x^{2}$ e o nivel de significância estabelecido foi de 0,05 .

\section{Resultados}

Foram identificadas 261 Declarações com CC, sendo 185 com uma só $C C$ registrada e 75 com duas $\mathrm{CC}$, produzindo, portanto, $335 \mathrm{CC}$. No total de Declarações (1.308), 453 tinham menos de 50 anos e 855 tinham 50 anos ou mais. Dentre os falecidos com registro de CC, 64 não haviam atingido os 50 anos, configurando assim uma "proporção de presença de causas contributárias" de 14,1\% (64/453) no estrato de menores de 50 anos; esta proporção foi significativamente inferior àquela detectada no

Tabela 1. Distribuição dos óbitos por cardiopatia chagásica, segundo a existência ou năo de causa contributária e faixa

\begin{tabular}{lccccc}
\hline \multicolumn{1}{c}{ Idade } & \multicolumn{2}{c}{$\leq 50$ anos } & \multicolumn{2}{c}{$>50$ anos } \\
$\begin{array}{l}\text { Causa } \\
\text { contributária }\end{array}$ & $n^{2}$ & $\%$ & $n^{2}$ & $\%$ & Total \\
\hline Ausente & 389 & 85,9 & 658 & 76,9 & 1.047 \\
Presente & 64 & 14,1 & 197 & 23,1 & 261 \\
\hline Total & 453 & 100,0 & 855 & 100,0 & 1.308 \\
\hline
\end{tabular}

$p<0,05$ 
Tabela 2. Causas contributárias das Declaraçōes de Óbito por cardiopatia chagásica. Estado de São Paulo, 1987.

\begin{tabular}{ll}
\hline Causas contributárias & $N^{2}$ \\
\hline Doenças não-infecto-parasitárias & \\
Embolias & 31 \\
Doenças Pulmonar Crônica & 28 \\
Desnutrição & 26 \\
Hipertensão Arterial & 26 \\
Acidente Vascular Cerebral & 24 \\
Insuficiência Renal & 20 \\
Arteriosclerose & 19 \\
Diabetes & 17 \\
Alcoolismo & 09 \\
Câncer & 08 \\
Outras não-infecto-parasitárias & 44 \\
Doenças infecto-parasitárias & 36 \\
Megas & 26 \\
Infecçöes (exceto chagas/ megas) & 21 \\
Pneumonia & 335 \\
\hline Total & \\
\hline
\end{tabular}

- Engloba megacolon, megaesôfago e megacolon mais megaesôfago

subgrupo com mais de 50 anos, que foi de $23,0 \%$ (197 casos com CC em 855 casos cuja CB era cardiopatia chagásica) (Tabela 1).
$\mathrm{Na}$ Tabela 2 encontram-se as distribuições das $335 \mathrm{CC}$ registradas nos 261 atestados. Como se nota, o eixo que foi valorizado foi o de dividir as doenças em infecto-parasitárias (DIP) e não infecto-parasitárias (NIP). Além disso, buscou-se também identificar subcategorias dentro destas duas categorias mais gerais, como por exemplo alcoolismo e pneumonia. É nítido o predomínio das doenças não infecto-parasitárias.

$\mathrm{Na}$ Tabela 3 listaram-se as primeiras $10 \mathrm{CC}$, em ordem decrescente de freqüência absoluta, em cada um dos agrupamentos de idade.

$\mathrm{Na}$ Tabela 4, calculou-se a "proporção de presença de uma causa contributária (específica)" para cada um dos grupos etários ( $<50 ; 50$ ou + ). Para o cálculo desses valores construiu-se uma proporção, que pode ser ilustrada tomando como exemplo as embolias. Pela Tabela 3 , verificou-se que ocorreram 10 casos de embolia no grupo etário abaixo de 50 anos; logo a "proporção de participação" da embolia nesse grupo foi de 10/453 (2,2\%), uma vez que os 453 falecidos abaixo de 50 anos constituem a "população exposta". O mesmo raciocínio é repetido tanto para o grupo de 50 anos ou mais, ou seja, 21 casos (Tabela 3 ) em 855 expostos, resultando numa "taxa de participação" de $25 \%$. Repetindo-se esse raciocinio para cada uma das situações, construiu-se a Tabela 4.

Nas Tabelas 5, 6 e 7 encontra-se a distribuição dos dois subgrupos - com CC (261) e sem CC

Tabela 3. Distribuição das principais causas contributárias das Declarações de Óbito por cardiopatia chagásica, segundo idade. Estado de São Paulo, 1987.

\begin{tabular}{llll}
\hline \multicolumn{1}{c}{$<50$ anos } & & \multicolumn{2}{c}{$\geq 50$ anos } \\
Causa contributária & Freq. & Causa contributária & Freq. \\
\hline Infecciosas (1² Cap. CID) & 10 & Megas & 28 \\
Embolias & 10 & Doença Pulmonar Crönica & 24 \\
Desnutrição & 8 & Hipertensão Arterial & 23 \\
Megas & 8 & Embolias & 21 \\
Alcoolismo & 6 & Acidente Vascular Cerebral & 21 \\
Insuficiência Renal & 5 & Desnutrição & 18 \\
Pneumonia & 5 & Arterioscierose & 17 \\
Doença Pulmonar Crônica & 4 & Pneumonia & 16 \\
Acidente Vascular Cerebral & 3 & Diabetes & 16 \\
Hipertensäo Arterial & 3 & Doenças Intecciosas (19 Cap. CID) & 16 \\
\hline
\end{tabular}

" Engloba megacolon, megaesôfago e megacolon mais megaesófago 
Tabela 4. Proporção de causas contributárias presentes nas Declarações de Óbito por cardiopatia chagásica, segundo grupo etário. Estado de São Paulo, 1987.

\begin{tabular}{|c|c|c|c|c|c|}
\hline \multirow[b]{2}{*}{ Causa contributária } & \multirow[t]{2}{*}{ Idade } & \multicolumn{2}{|c|}{$<50$ anos } & \multicolumn{2}{|c|}{$\geq 50$ anos } \\
\hline & & $N^{2}$ & Proporção* & $\mathrm{N}^{9}$ & Proporção** \\
\hline Ac. Vasc. Cerebral & & 3 & $0,7^{(1)}$ & 21 & $2,5^{(1)}$ \\
\hline D. Pulm. Crônica & & 4 & $0,9^{(2)}$ & 24 & $2,8^{(2)}$ \\
\hline Desnutriçăo & & 8 & 1,8 & 18 & 2,1 \\
\hline Embolia & & 10 & 2,2 & 21 & 2,5 \\
\hline Hip. Arterial & & 3 & $0,7^{(3)}$ & 23 & $2,7^{(3)}$ \\
\hline Infecção & & 10 & 2,2 & 16 & 1,9 \\
\hline Insufic. Renal & & 5 & 1,1 & 15 & 1,8 \\
\hline Megas ${ }^{\star \star *}$ & & 8 & 1,8 & 28 & 3,3 \\
\hline
\end{tabular}

(*) denominador 453

$(* *)$ denominador 855

* *Engloba megacolon, megaesôfago e megacolon mais megaesôfago

(1)(2)(3) $p<0,05$

Tabela 5. Distribuição dos óbitos por cardiopatia chagásica, segundo a existência ou não de causa contributária e faixa etária. Estado de São Paulo, 1987.

\begin{tabular}{|c|c|c|c|c|c|c|c|c|}
\hline \multirow[b]{2}{*}{ Causa Contributária } & \multirow[t]{2}{*}{ Idade } & \multicolumn{2}{|c|}{ até 39 anos } & \multicolumn{2}{|c|}{40 a 59 anos } & \multicolumn{2}{|c|}{60 e mais } & \multirow[t]{2}{*}{ Total } \\
\hline & & $\mathrm{N}^{\mathrm{e}}$ & $\%$ & $\mathrm{~N}^{\mathrm{e}}$ & $\%$ & $N^{2}$ & $\%$ & \\
\hline Ausente & & 191 & 18,0 & 489 & 47,0 & 367 & 35,0 & 1.047 \\
\hline Presente & & 29 & 11,1 & 93 & 35,6 & 139 & 53,3 & 261 \\
\hline Total & & 220 & 16,8 & 582 & 44,5 & 506 & 38,7 & 1.308 \\
\hline
\end{tabular}

$p<0,05$

(1.047) - segundo as variáveis idade, sexo e local de atendimento. Dentre as 3 variáveis, a idade foi a única que apresentou uma associação significativa $(p<0,05)$.

\section{Discussão}

As variáveis relacionadas podem ser classificadas em dois tipos: aquelas cujo resultado admite algum grau de previsibilidade, como é o caso da variável "idade", e aquelas que não permitem essa previsibilidade, no caso o sexo e local da residência.

A previsibilidade se baseia no seguinte racio- cício: pode-se esperar que pacientes mais idosos apresentem uma maior proporção de $\mathrm{CC}$, em função do quadro de morbidade crescente com a idade. $\mathrm{E}$ de fato, esse dado ocorreu (Tabela 5).

Entendeu-se que a confirmação do comportamento dessa variável representa um indicador indireto - da consistência das informações existentes nas 1.308 Declarações analisadas. Daí o valor de se ter um indicador desta qualidade, ainda que indireto.

A hipótese do presente estudo, em relação a questão da qualidade, é a da existência de um subregistro das $\mathrm{CC}$. Nesse contexto é que se tornam 
Tabela 6. Distribuição dos pacientes falecidos por cardiopatia chagásica, segundo a presença ou não de causa contributária e sexo. Estado de São Paulo, 1987.

\begin{tabular}{lcccccc}
\hline & Sexo & Masculino & & Feminino & Total \\
Causa Contributária & N2 & $\%$ & No & $\%$ & \\
\hline Presente & 172 & 65,9 & 89 & 34,1 & 261 \\
Ausente & 666 & 63,9 & 381 & 36,3 & 1.047 \\
\hline Total & 838 & 64,1 & 470 & 35,9 & 1.308 \\
\hline
\end{tabular}

$p>0,05$

Tabela 7. Distribuição dos pacientes falecidos com cardiopatia chagásica, segundo a presença ou não de causa contributária e local de atendimento. Estado de Sāo Paulo, 1987.

\begin{tabular}{|c|c|c|c|c|c|}
\hline \multirow{2}{*}{ Causa Contributária Local de atendimento } & \multicolumn{2}{|c|}{ Grande São Paulo } & \multicolumn{2}{|c|}{ Interior } & \multirow[t]{2}{*}{ Total } \\
\hline & $\mathrm{N}^{2}$ & $\%$ & $\mathrm{~N}^{2}$ & $\%$ & \\
\hline Presente & 103 & 39,5 & 158 & 60,5 & 261 \\
\hline Ausente & 390 & 37,2 & 657 & 62,8 & 1.047 \\
\hline Total & 493 & 37,7 & 815 & 62,3 & 1.308 \\
\hline
\end{tabular}

$P>0,005$

úteis os dados relativos à idade na medida em que indicam grau da consistência no registro das $\mathrm{CC}$.

No caso das variáveis não previsíveis constatou-se que não houve diferença na distribuição segundo sexo (Tabela 6) e que também não houve diferença, estatisticamente significante, nas proporções dos atestados com CC na Grande São Paulo e no interior (Tabela 7). Essas variáveis se inserem na linha de pesquisa que busca identificar os determinantes da presença das $\mathrm{CC}$ no grupo de chagásicos estudado.

Dois outros aspectos devem ser ressaltados. $\mathrm{O}$ primeiro é a participação importante, nas causas contributárias (CC), tanto das DIP como das NIP. A importância desta última no presente material pode ser confirmada a partir da sua predominância quantitativa (Tabela 2): 252 (75,2\%) entre as 335 causas assinaladas fazem parte do grupo de doenças NIP. O subgrupo formado pelas categorias arteriosclerose, acidente vascular cerebral, câncer, diabetes, doença pulmonar crônica, embolia, hipertensão arterial e insuficiência renal - doenças denominadas de crônico-degenerativas - englobam $68,7 \%$ das 252 causas desse grupo.

Já a identificação da importância da participação das DIP requer uma análise mais contextualizada. Inicialmente é necessário considerar que as DIP estão distribuídas em 3 grupos (Tabela 2): os 83 registros de doenças infecciosas $(24,8 \%$ em relação ao total) compreendem 36 relatos de megas, 21 de pneumonia e 26 registros de "doenças infecciosas". Pode-se argumentar que dado que a existência de um mega é parte do quadro de um doente chagásico, esse evento não deve ser destacado, mesmo tendo sido registrado como contributária de um atestado cuja causa básica foi cardiopatia chagásica. Admitindo esse argumento mais conservador, comparouse esse percentual residual (14\% quando se excluem os megas) com os dados do Anuário Estatístico do Estado de São Paulo ${ }^{4}$, no ano de 1987 , lembrando 
que foi considerada, naquela publicação, apenas a causa básica de óbito: em 157.460 óbitos de maiores de 15 anos, $4,7 \%$ foram de pneumonia e $3,8 \%$ de causas classificáveis no $1^{\circ}$ capítulo da CID, excluindo doença de Chagas. Essa mesma tendência é constatada quando se analisa as causas múltiplas de morte no Estado de São Paulo ${ }^{3}$ : as doenças infecciosas estão presentes nos chagásicos, numa maior proporção do que em outros grupos.

Esses dados - relativos às DIP e NIP - permitem afirmar que para esse grupo populacional que faleceu de cardiopatia chagásica (que é uma parte da população chagásica do Estado de São Paulo) são válidas as constatações de Laurenti ${ }^{6}$, que afirmou, já em 1972, a presença conjunta destes eventos (DIP e NIP) no quadro de morbi-mortalidade. O presente estudo, além de confirmar essa tendência geral assinalada por Laurenti ${ }^{6}$ e reafirmada em outro trabatho ${ }^{10}$, permite também apontar para a possibilidade de que a participação das doenças infecciosas, como CC no grupo de falecidos cuja causa básica é cardiopatia chagásica, seja superior àquela existente na maioria dos diversos grupos populacionais do Estado. É claro que para analisar essa hipótese será necessário examinar a participação das doenças infecciosas como CC em outros grupos específicos de causas básicas, análise que transcende aos objetivos da presente pesquisa. Registre-se, apenas como contribuição ao assunto, que na revisão bibliográfica que se efetuou não foi encontrado esse tema analisado em maior profundidade.

O segundo aspecto é a participação dessas CC em dois grupos de falecidos, divididos segundo a idade. A análise da Tabela 3 permite afirmar que: a) embolias e megas estão presentes entre as 5 primeiras causas nos 2 subgrupos de idade, afirmando assim a sua importância; b) as doenças infecciosas passam de primeiro lugar no grupo mais jovem para o décimo lugar no grupo mais idoso; c) o alcoolismo, que não consta da lista de grupo mais idoso, até décima colocação, ocupa o quinto lugar no grupo abaixo de 50 anos.

Apesar das diferenças de colocação das diversas causas- quando se constrói a escala com números absolutos e considerando somente a classificação interna dentro da faixa etária, como verifica-se na Tabela 3 - quando se calculou a "proporção da presença de uma causa contributária" específica em cada grupo etário, essas proporções apresentaram, em muitas situações, valores semelhantes nos dois grupos etários (Tabela 4).

É bastante ilustrativo o caso das doenças infecciosas: apesar da disparidade de posição dessas doenças (Tabela 3 ) as "proporções de presença" foram de $2,2 \%$ e $1,9 \%$ (Tabela 4 ), sendo a diferença não significativa do ponto de vista estatístico. Isso significa que a probabilidade - tomada aqui no sentido de "risco" - da presença de uma doença infecciosa como CC é semelhante em ambos subgrupos ( $<50$ e $50 \mathrm{ou}+$ ); considerando, no entanto, uma escala com números absolutos, a doença infecciosa ocupa o primeiro lugar num subgrupo e décimo noutro subgrupo.

Esses dados ilustram a importância e a pertinência de se trabalhar com as duas perspectivas número absoluto e proporção, considerando os diferentes significados desses elementos.

Como colocação geral, pode-se afirmar que existe uma relevante presença de CC nos falecidos por cardiopatia chagásica ( $20 \%$ dos atestados), e que a análise dessas causas, bem como de variáveis selecionadas da Declaração de Óbito, permitem aduzir contribuições para o conhecimento do perfil do paciente chagásico.

WANDERLEY, D.M.V \& LITVOC, J. [Chagas disease as principal cause of death in area of southeastern Brazil: presence of contributory causes].Rev. Saude Pública, 28:69-75, 1994. Death certificates of all persons who died in 1987 in the State of S.Paulo, Brazil and which presented Chagas' disease as the principal cause of death, were studied with a view to analysing the existing additional information available as to contributory causes. After a direct reading of the 1,308 death certificates, the contributory causes were identified and registered. They were mentioned in 261 $(20 \%)$ of the certificates, 185 of them presenting only one, and 75 two of them. The 6 more frequent contributory causes were: "megas", embolism, chronic pulmonary disease, infections (other than Chagas' disease), arterial hypertension and malnutrition. When analysisng the presence of the contributory causes in two groups - persons of less than 50 years old, and those older than 50 a higher proportion of them was observed in the older group and a distinct profile of causes was found for each group. No statistic association was observed between contributory causes and sex or site of residence.

Keywords: Trypanosomiasis, South American, mortality. Cause of death. 


\section{Referências Bibliográficas}

1. ALMEIDA, H. Estudo da morbidade no municipio de São Paulo. São Paulo, 1983. [Dissertação de Mestrado - Faculdade de Medicina da USP].

2. CORTEZ JR., L. de S. Mortalidade, segundo causas múltiplas no municipio de Botucatu. Sāo Paulo, 1976. [Tese de Doutorado - Faculdade de Ciências Médicas da UNICAMP].

3. FUNDAÇÃO SEADE. Anuário estatístico do Estado de São Paulo: 1986. São Paulo, 1987.

4. FUNDAÇÃO SEADE. Anuário estatístico do Estado de São Paulo: 1989. São Paulo, 1991.

5. JATENE, A. D. et al. Assistência à saúde na Região Metropolitana de São Paulo - Brasil. In: Reunião Regional/ Oms/ops sobre Desenvolvimento de Serviços de Saúde nas Grandes Cidades da América Latina, Washington, 1981.

6. LAURENTI, R. \& SILVEIRA, M.H. Causas múltiplas de morte. Rev. Saúde Pública, 6: 97-102, 1972.

7. LAURENTI, R. Causas múltiplas de morte. São Paulo, 1973. [Tese de Livre Docência - Faculdade de Saúde Pública da USP].

8. LAURENTI, R. et al. Estatística de saúde. São Paulo, EPU/EDUSP, 1985.

9. LITVOC, J.; WANDERLEY, D.M.V.; ARANHA CAMARGO, L.M. Mortalidade por doença de Chagas no Estado de São
Paulo: subsidios para o planejamento da assistência ao chagásico. Rev. Saúde Pública, 26: 59-65, 1982.

10. POSSAS, C. Epidemiologia e sociedade; heterogeneidade estrutural e saúde no Brasil. São Paulo, Hucitec, 1989.

11. ORGANIZAÇÃO MUNDIAL DA SAÚDE. Manual de classificação internacional de doenças, lesōes e causas de óbitos. 9 rev., 1975. São Paulo - Centro da OMS para a Classificação de Doenças em Português, 1978.

12. SALA, A. Avaliaçāo em serviço de atenção primária à saúde: instrumentos de análise de processo de trabalho médico. São Paulo, 1988. [Dissertação de Mestrado - Faculdade de Medicina da USP].

13. SANTO, A.H. Causas múltiplas de morte: formas de apresentação e métodos de análise. São Paulo, 1988. [Tese de Doutorado - Faculdade de Saúde Pública da USP].

14. SECRETARIAMUNICIPAL DE SAÚDE. Diagnóstico de saúde no municipio de São Paulo. São Paulo, 1990. [mimeografado].

15. SCHRAIBER, L.B.; org. Programação em saúde hoje. São Paulo, Hucitec, 1990.

Recebido para publicação em 20.5.1993 Reapresentado em 26.10.1993 Aprovado para publicação em 30.11 .1993 